La reproduction sexuée implique une alternance entre phase haploide et phase diploïde. La méiose fait passer les cellules à l'état haploìde. La fécondation ou la conjugaison restaure la diploïdie. Cet article tente d'expliquer deux aspects essentiels de la reproduction sexuée. Premièrement, le zygote réunit deux gamètes de phénotype distinct. Deuxièmement, la différence entre gamètes se traduit très souvent par un dimorphisme de taille. Cette particularité est appelée anisogamie. Elle est à l'origine de la différenciation des organismes en mâles et femelles.

L'union des gamètes a une exigence fondamentale: elle doit limiter à deux le nombre de cellules qui fusionnent, sinon la méiose ne pourra pas rétablir l'haploïdie. Cela rend nécessaire la diversification des gamètes en deux types, différant de telle façon que le zygote acquière des propriétés nouvelles et ne puisse pas fusionner avec une autre cellule. L'anisogamie pourrait découler d'une propriété très ancienne des organismes sexués, qui est de favoriser le rapprochement des gamètes en leur faisant produire des substances attractives. Ce système fonctionne de façon optimale si les gamètes appartiennent à deux catégories distinctes. Les uns sont immobiles et envoient le signal attractif. Les autres répondent au signal en se déplaçant vers les cellules émettrices. On peut penser que la sélection naturelle a favorisé deux types de mutations: celles qui accroissent la taille des gamètes immobiles et celles qui diminuent la taille des gamètes mobiles. Ces modifications ont des avantages évidents. Les cellules plus grandes auront un pouvoir attractif accru et donneront des zygotes plus aptes à survivre. Les cellules plus petites seront plus faciles à mouvoir et à produire en grand nombre, ce qui accroît les probabilités de rencontre avec un partenaire de type

\title{
L'origine et l'évolution de la reproduction sexuée
}

\author{
Herman Denis, Alain Collenot
}

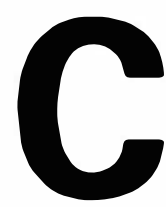

hez l'homme et la plupart des animaux, la reproduction occupe une place à part parmi toutes les fonctions corporelles. En général, un individu isolé est capable de survivre sans l'aide de ses congénères. Mais pour se reproduire, il doit collaborer avec un autre individu. Les animaux consacrent à la reproduction un temps et une énergie considérables. Il suffit pour s'en convaincre d'observer les oiseaux en train de nidifier, ou encore d'examiner un oursin ouvert à l'étal d'un écailler: le test de l'animal est envahi par les gonades, à tel point que celles-ci dépassent en volume tous les autres organes réunis.

Dans l'imaginaire humain, la reproduction est indissolublement liée à la sexualité. Reproduction et sexualité sont un terrain fertile sur lequel poussèrent mythes et légendes. En remontant par la pensée les générations qui nous ont précédés, on finit par buter sur quelque événement fondateur, dont on connaît deux versions principales: ou bien les sexes reflètent une dualité fondamentale de l'univers, et leur existence est aussi ancienne que le monde lui-même; ou bien les sexes résultent d'une brisure secondaire dans une création qui était une à l'origine [1]. Le yin et le yang du taoisme appartiennent au premier type de mythologie [1]. Le mythe biblique d'Adam et Eve se range dans la seconde catégorie [1]. Dans les deux cas, la reproduction sexuée peut s'interpréter comme un effort des procréateurs pour unir ou réu- nir les forces opposées qui se conjuguent pour créer un nouvel être.

De nos jours, les scientifiques continuent à réfléchir sur l'origine de la sexualité et de la reproduction sexuée [2-4]. Ces deux concepts recouvrent des réalités différentes. On qualifie de sexuels les phénomènes naturels impliquant la formation d'un nouvel individu dont les gènes dérivent de plus d'un parent [5]. La reproduction sexuée ne concerne que les espèces dont toutes ou certaines cellules passent alternativement d'une phase diploïde à une phase haploïde.

Cela fait que des organismes tels que les bactéries possèdent une sexualité, sans pour autant se reproduire de façon sexuée.

Nous nous intéressons surtout aux protistes, qui sont des eucaryotes unicellulaires, et aux métazoaires, qui dérivent probablement des protistes. La plupart de ces organismes recourent à la reproduction sexuée, soit de façon occasionnelle, soit de façon permanente. On peut donc penser que ce mode de propagation s'instaura très précocement au cours de l'évolution des protistes, et bien avant l'apparition des métazoaires.

Par suite du passage au stade multicellulaire, la reproduction sexuée n'a pas la même importance pour les protistes et pour les métazoaires. Cette différence se marque de deux façons. En premier lieu, la reproduction sexuée n'est pour les protistes qu'un parmi deux modes de propagation, l'autre étant le mode végé- 
tatif, qui ne comporte que des mitoses. Chez la majorité des métazoaires, la reproduction sexuée est le mode de propagation principal, sinon exclusif. En second lieu, la reproduction sexuée expose beaucoup de protistes à un danger : celui de ne pas rencontrer de partenaire sexuel, ce qui les prive le plus souvent de descendance. Pour les métazoaires, le risque est moindre. La plupart d'entre eux ont au cours de leur vie plusieurs occasions de se reproduire, en émettant à chaque fois de nombreux gamètes.

\section{Alternance entre phases haploide et diploïde}

Puisque la reproduction sexuée est liée à l'alternance entre une phase haploïde (ou haplophase) et une phase diploïde (ou diplophase), il convient de s'interroger sur l'origine de cette alternance. Ce phénomène rythme le cycle vital de presque tous les eucaryotes actuels. Des exceptions se rencontrent dans quelques groupes de protistes (euglènes, amibes), qui sont en permanence haploïdes et se reproduisent de façon végétative, sans jamais subir la méiose [6]. Parmi les métazoaires, on ne connaît pas d'espèce strictement haploïde.

Il y a chez les protistes de grandes variations dans la durée relative des phases haploïde et diploïde. A cet égard, les protistes peuvent se ranger en trois catégories (figure 1) [6, 7]. Certains d'entre eux sont des haplontes; la méiose a lieu après la fusion des gamètes, si bien que seul le zygote est diploïde. Chez d'autres protistes (les foraminifères), le zygote ne subit pas tout de suite la méiose; la fusion des gamètes est suivie par une génération diploïde, après quoi la méiose instaure une génération haploïde. Les protistes de la troisième catégorie sont des diplontes. Chez eux, la méiose précède la formation des gamètes.

On retrouve chez les organismes multicellulaires les trois catégories que l'on distingue parmi les protistes (figure 1). Les protistes coloniaux (volvocales) sont des haplontes [8]. C'est le zygote qui subit la méiose. Certains végétaux (algues, champi$\mathrm{m} / \mathrm{s} n^{\circ} 12$ vol. 9, décembre 93 gnons, fougères, mousses...) se rangent dans la deuxième catégorie: leur cycle vital comporte une alternance entre générations haploïde et diploïde [9]. Les métazoaires appartiennent à la troisième catégorie, puisque chez eux la méiose se produit pendant la gamétogenèse. Sauf exceptions très rares, l'œuf, l'embryon et l'adulte sont diploïdes. Du point de vue évolutif, il est clair que l'alternance des phases s'est imposée chez des eucaryotes très primitifs, et maintenue dans leur descendance. Il est difficile de préciser quelle position la méiose occupait dans le cycle vital des protistes archaïques (figure 1). Une position zygotique ou intermédiaire semble la plus probable, ce qui revient à dire qu'initialement la gamétogenèse se déroulait durant l'haplophase [6]. On conçoit aisément que la phase diploïde ait pu s'allonger ou se raccourcir pendant l'évolution des protistes: il suffit qu'après l'union des gamètes le nombre de divisions végétatives augmente ou diminue.

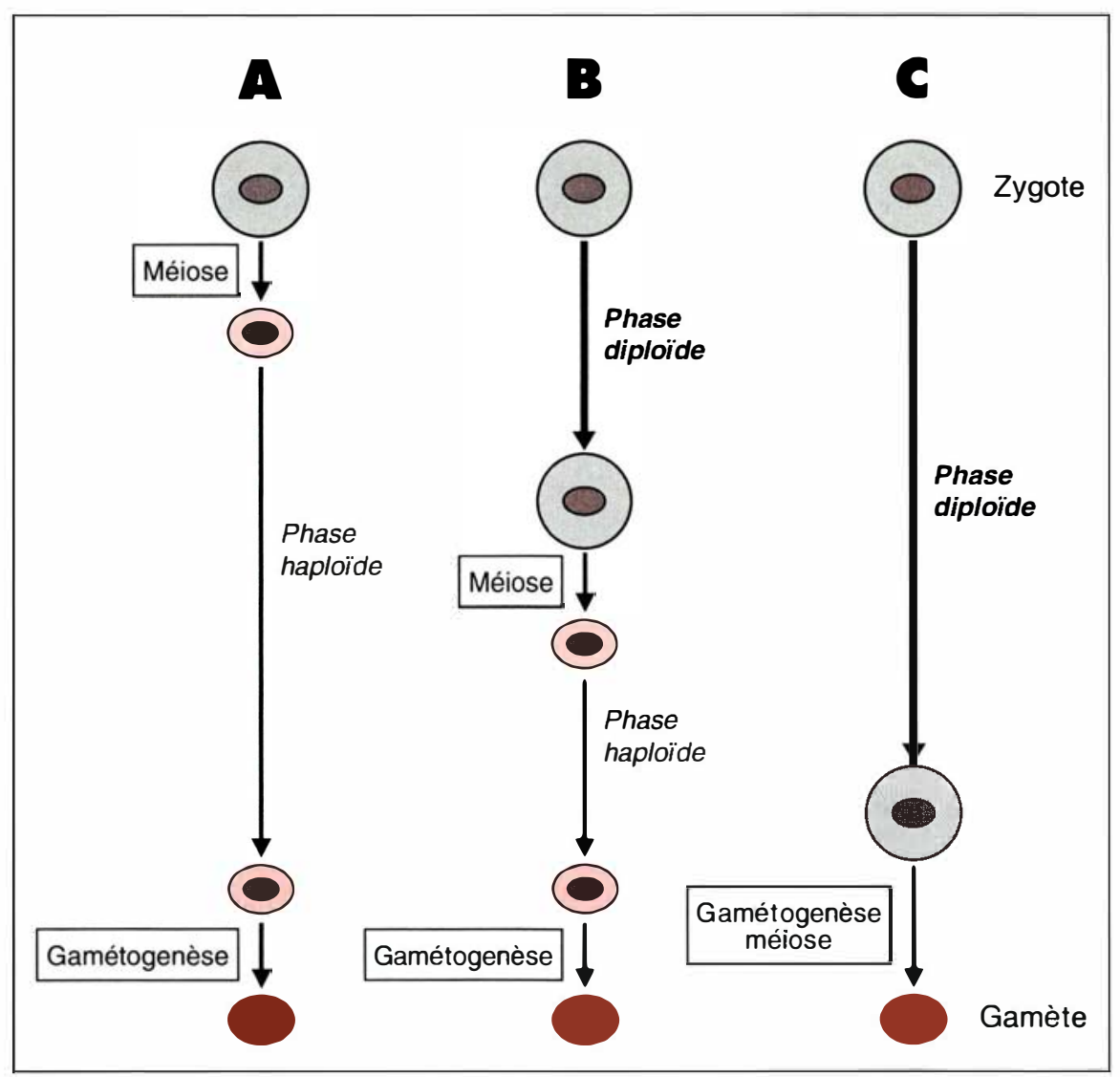

Figure 1. Alternance des phases haploïde et diploïde chez les protistes. Le cycle vital fait alterner des périodes de multiplication végétative avec des périodes de reproduction sexuée. La multiplication végétative se fait par mitose. La reproduction sexuée implique une gamétogenèse, c'est-à-dire la formation de gamètes haploïdes, puis la fusion des gamètes en un zygote diploïde. La gamétogenèse peut être découplée de la méiose car celle-ci occupe une position variable dans le cycle vital. Chez les haplontes (A), la méiose suit immédiatement la formation du zygote. La période végétative est donc haploïde. Chez d'autres protistes (B), la méiose est retardée par rapport à la formation du zygote. Elle divise la période végétative en deux phases: diploïde, puis haploïde. Les diplontes (C) subissent la gamétogenèse et la méiose tout à la fin de la période végétative. Les métazoaires sont des diplontes. Chez eux, la méiose se produit pendant la gamétogenèse, si bien que seuls les gamètes sont haploïdes. 


\section{Origine}

\section{de la diploïdie}

Comment s'est fait le passage de l'haploïdie à la diploïdie? Le mécanisme le plus simple implique une seule cellule, qui double sa garniture chromosomique. On peut imaginer une division nucléaire (caryocinèse) suivie d'une fusion des noyaux, en l'absence de division cytoplasmique (cytocinèse) [5]. L'endomitose offre une autre possibilité de diploïdisation: la cellule réplique ses chromosomes, mais son noyau ne se divise pas. Un tel processus se produit chez certains protistes : flagellés, radiolaires, ciliés [6, 7].

Un mécanisme moins orthodoxe fait intervenir deux cellules. On suppose qu'un protiste haploïde en avale un autre, sans que l'ingestion cause la destruction du noyau de la proie [5]. Un tel phénomène s'observe chez certains héliozoaires actuels [10]. On obtient ainsi une cellule binucléée ou plurinucléée (hétérocaryon), qui peut redevenir uninucléée par simple partition du cytoplasme. $\mathrm{Si}$ les conditions du milieu varient de façon cyclique, une alternance s’instaure entre états plurinucléé et uninucléé. Quand les conditions deviennent défavorables, l'état plurinucléé est avantagé, et ce pour deux raisons. Réunissant des noyaux de génotypes différents, l'hétérocaryon pourra bénéficier de complémentations efficaces. S'étant agrandi, il aura un rapport surface/volume plus avantageux et opposera ainsi une meilleure résistance, notamment à la dessication. Lorsque les conditions s'améliorent, c'est la vitesse de multiplication qui est privilégiée. L'hétérocaryon se scinde et revient à l'état uninucléé parce que ce dernier est mieux adapté à une division cellulaire rapide.

Dans une étape ultérieure, on peut envisager une fusion entre les noyaux coexistant dans l'hétérocaryon. On obtient ainsi des cellules diploïdes ou polyploïdes. Au lieu d'une variation cyclique dans le nombre de noyaux, nous avons maintenant une variation de ploïdie, imposée par le milieu. Lorsque les fusionnent et deviennent polyplödes.

Nous privilégions une telle origine pour la diploïdie. Nous y voyons certains avantages. Le cannibalisme invoqué préfigure l'union des gamètes, tout en associant la diploïdisation avec une fusion cellulaire. Quant au milieu, il continue de nos jours à jouer un rôle important dans le déroulement du cycle vital. Chez de nombreux organismes (divers protistes, certains champignons et algues), c'est la privation de nourriture qui déclenche la reproduction sexuée, donc la fusion cellulaire, après une période de multiplication rapide [7, 11].

\section{Origine de la méiose}

Pour retourner à l'état haploïde, une cellule polyploïde doit faire le tri entre plusieurs assortiments de chromosomes, pour que les noyaux fils gardent une garniture chromosomique équilibrée. La méiose fait un tel tri, mais elle ne peut pas répartir les chromosomes en plus de deux lots.

Il est très malaisé de reconstituer l'origine et l'évolution de la méiose. Il se pourrait que la méiose apparût avant la reproduction sexuée, comme un moyen de diminuer la polyploïdie engendrée par endomitose $[12,13]$. De fait, on connaît des flagellés dont la ploïdie varie de façon cyclique, s'accroissant par endomitose et se réduisant par un processus qui ressemble à la méiose [12, 14]. Mais ce processus pourrait être une modification de la méiose, plutôt qu'un phénomène général à partir duquel celle-ci aurait pu se construire.

Nous pensons que la méiose apparut progressivement, à la suite d'une longue période de tâtonnements. Il est possible que ces tentatives se soient déroulées dans des cellules plurinucléées, provenant de fusions multiples. Peu à peu, la méiose s'associa à une fusion entre noyaux provenant de deux cellules, et se cantonna à un tri entre deux assortiments de chromosomes. Pendant ces essais, le déclenchement de la méiose devait rester lié à une amélioration dans les conditions du milieu, qui favorisait la multiplication de cellules haploïdes.

Concernant l'origine de la méiose, un point fait l'accord général: la méiose dérive de la mitose [11]. Les organismes actuels gardent des traces de cette filiation. Les deux processus utilisent le même appareil cytosquelettique. Tous ou presque tous les gènes qui gouvernent le déroulement de la mitose sont aussi impliqués dans la méiose [15, 16].

\section{Les deux types de méiose}

Peut-on savoir si la méiose apparut à une seule reprise ou à plusieurs reprises au cours de l'évolution? Chez les protistes, la méiose se fait suivant deux modalités (figure 2). Ia méiose de type 1 ne comporte qu'une seule division. Elle n'est pas précédée par la duplication des chromosomes. Au cours de la prophase, les chromosomes homologues s'apparient, sans apparemment se recombiner [6]. I'unique division distribue un homologue de chaque paire entre le's deux cellules filles. Une méiose de cette nature a été décrite chez les sporozoaires, qui sont des haplontes, et chez certains flagellés haplontes et diplontes $[6,7$, $12,17,18]$. Cependant, il faut considérer ces observations avec prudence. Ians aucun groupe de protistes, l'existence d'une méiose en une division n'est démontrée conjointement par trois types d'étude : cytologique, génétique (analyse des produits de la méiose) et moléculaire (mesure du contenu en $\mathrm{ADN}$ des cellules).

Une méiose plus complexe est pratiquée par les autres protistes haplontes et diplontes, ainsi que par tous les organismes multicellulaires. La méiose de type 2 comprend deux divisions (figure 2). Les chromosomes commencent par se dupliquer. Pendant la prophase de la première division, les homologues s'apparient en tétrades, puis se recombinent. Les deux divisions successives séparent les tétrades en quatre chromatides, dont les quatre cellules filles reçoivent un exemplaire. En ce qui concerne la redistribution des gènes entre les cellules, la méiose de type 2 est plus efficace 


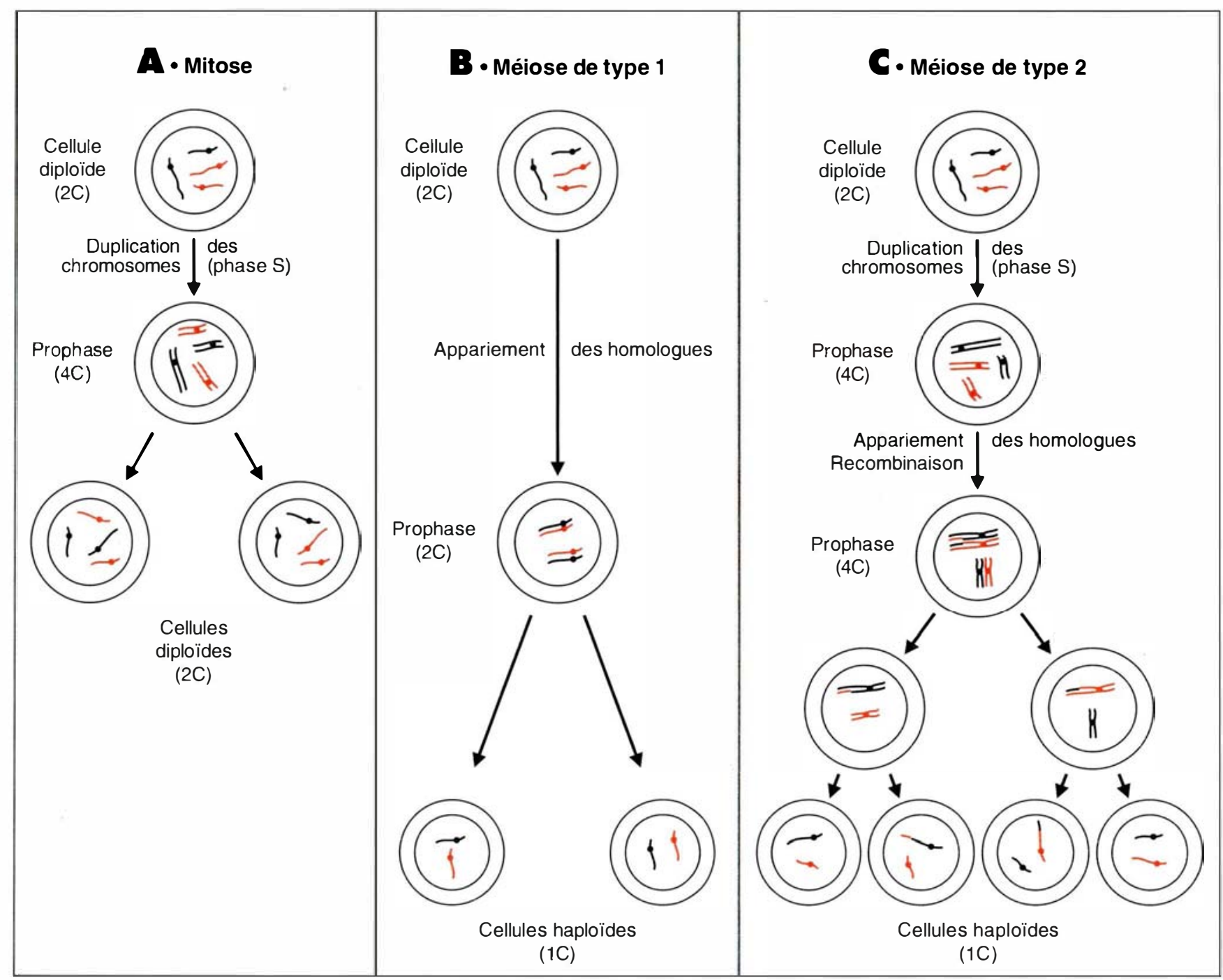

Figure 2. Comparaison entre la mitose et la méiose. Le mécanisme de la mitose (A) est le même, que la cellule soit haploïde ou diploïde. La méiose se déroule suivant deux modalités principales. La méiose de type 1 (B) s'observe chez certains protistes. Elle comporte une seule division. Sans s'être au préalable dupliqués, les chromosomes homologues s'apparient en dyades. Ils ne semblent pas subir de recombinaison. La méiose de type 2 (C) est pratiquée par la plupart des protistes et les organismes multicellulaires. Elle comprend deux divisions successives. Les chromosomes se dupliquent, s'apparient par paires homologues pour former des tétrades, puis se recombinent. La première division sépare chaque tétrade en deux paires de chromatides, qui restent unies par leur centromère non scindé. La seconde division sépare les chromatides individuelles.

que la méiose de type 1 , puisque la recombinaison permet un échange de gènes entre les chromosomes homologues (figure 2).

Pour expliquer l'origine des deux formes de méiose, une hypothèse simple vient à l'esprit: l'une et l'autre ont évolué indépendamment à partir de la mitose. Un argument appuie cette conjecture. La mitose et la méiose de type 2 ont une propriété commune : elles séparent des $m / s \quad n^{\circ} 12$ vol. 9 , décembre 93 chromosomes dupliqués (figure 2). Par contre, la méiose de type 1 unit, puis disjoint des chromosomes non dédoublés.

Une seconde hypothèse consiste à postuler une filiation entre la méiose de type 1 et la méiose de type 2. Trois considérations soutiennent ce point de vue. En premier lieu, la méiose en une seule division est plus simple que la méiose en deux divisions. En deuxième lieu, il est probable que la méiose apparut comme le prolongement - immédiat ou différé - d'une fusion cellulaire. Apparemment, rien n'imposait qu'elle fût précédée d'une duplication des chromosomes. La méiose archaiqque n'aurait donc comporté qu'une seule division. En troisième lieu, il y a un trait commun entre les deux formes de méiose, ce qui milite en faveur d'une dérivation de l'une à partir 
de l'autre. Le point commun est la présence de complexes synaptonémaux, qui sont bien reconnaissables au microscope électronique. Dans les cellules qui subissent la méiose de type 2 , ces formations maintiennent unis les chromosomes homologues pendant le stade pachytène de la prophase I [19]. De tels complexes ont été décrits chez un sporozoaire [20], dont la méiose est censée ne comporter qu'une seule division [6].

On est ainsi amené à concevoir une conversion en deux étapes de la mitose en méiose de type $2:$ mitose $\rightarrow$ méiose de type $1 \rightarrow$ méiose de type 2 [6]. La première étape implique deux changements majeurs (figure 2). Premièrement, il faut apparier les chromosomes homologues. Deuxièmement, il faut diviser les cellules sans dupliquer les chromosomes, donc dissocier la réplication de l'ADN et la division cellulaire. Cela réclame un gain de fonctions par rapport au mécanisme qui, dans le cycle cellulaire habituel, subordonne le démarrage de la mitose à la réplication complète de l'ADN [21]. La seconde étape requiert une modification importante: non seulement ce sont des chromosomes dupliqués qui s'apparient dans la méiose de type 2 , mais les chromatides restent unies par un centromère non scindé. De la sorte, deux chromatides à la fois migreront vers les pôles de la cellule au cours de la première division.

Il est difficile de faire un choix entre les deux hypothèses énoncées plus haut. Quel que soit le point de vue adopté, on doit considérer l'origine de la méiose comme un phénomène complexe. C'est ce que montre la distribution parmi les protistes de la méiose de type 1 : celleci s'observe dans quelques groupes assez éloignés $[6,7,12,17,18]$. Si l'on tient pour vraie la première hypothèse, il faut admettre que des lignées différentes de protistes transformèrent la mitose en l'un ou l'autre type de méiose. La seconde hypothèse conduit à supposer que des événements évolutifs indépendants convertirent dans des lignées distinctes de protistes la méiose de type 1 en méiose de type 2. La dans les groupes où cette conversion n'a pas eu lieu.

\section{La différenciation sexuelle}

Les protistes instaurent la phase diploïde par deux moyens différents: la conjugaison ou la syngamie [22]. Dans le premier cas, les cellules s'accolent, échangent un noyau, puis se séparent. Dans le second cas, les gamètes fusionnent en une cellule unique. Parmi les protistes actuels, seuls les ciliés pratiquent la conjugaison. Nous pensons que c'est à la syngamie que recouraient les protistes archaïques. En effet, nous considérons la syngamie comme l'événement fondateur de la diploïdie.

Il est possible que, sous sa forme la plus primitive, la syngamie autorisait l'union de cellules identiques. Il en résulte un grave inconvénient. Le zygote a les mêmes propriétés que les gamètes. En particulier, sa membrane porte les mêmes molécules, grâce auxquelles les gamètes peuvent se reconnaître, adhérer l'un à l'autre, puis fusionner. Le zygote ne pourra donc empêcher d'autres cellules de se joindre à lui (figure 3). Ces unions multiples engendrent des cellules incapables de se convertir en gamètes. En effet, la méiose ne peut pas distribuer les chromosomes en plus de deux assortiments. Les protistes ont résolu ce problème en produisant des gamètes de deux phénotypes distincts, qui s'unissent en un zygote inapte à toute nouvelle fusion [7]. Pour ce faire, ils utilisent deux systèmes différents : le système dioïque (dioécie) ou le système monoïque (monoécie).

Les protistes dioïques possèdent deux types sexuels et produisent des gamètes de deux génotypes (+ ou -). Le système dioïque le plus simple qui se puisse concevoir fait intervenir un groupe de gènes dont l'activation déclenche la gamétogenèse (figure 4). Les produits de ces gènes rendent les gamètes aptes à fusionner, mais suppriment cette aptitude dans le zygote. L'un des gènes joue un rôle clé dans la diversification des cellules en deux types. Ce gène est appelé déterminateur (d). Il existe sous deux formes alléliques (+ ou -).

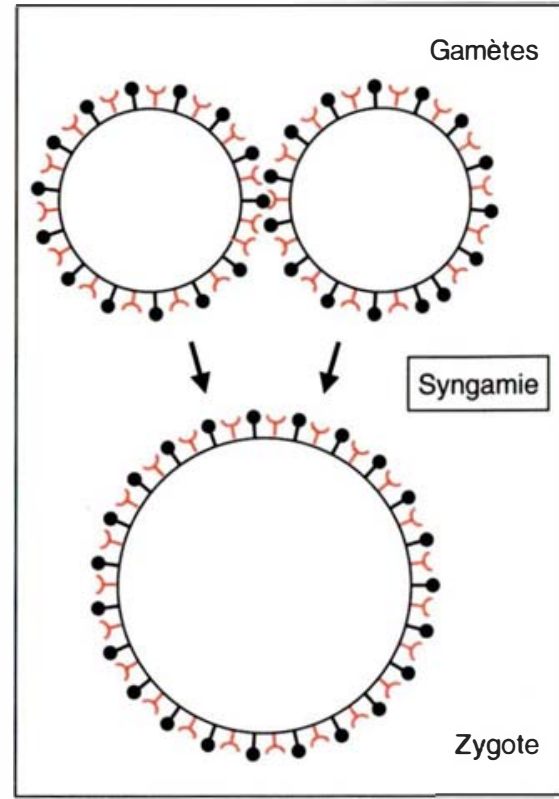

Figure 3. Fusion entre gamètes identiques. Pour fusionner, les gamètes doivent se reconnaitre et adhérer l'un à l'autre grâce à des molécules qui garnissent leur surface. Ces molécules peuvent être concentrées en un endroit privilégié de la surface cellulaire, tel que le flagelle. En s'agglutinant par leur(s) flagelle(s), les cellules se préparent à la fusion. Si deux cellules fusionnent parce qu'elles portent des molécules de reconnaissance complémentaires, le zygote possédera les mêmes propriétés que ses progéniteurs. II ne pourra pas empêcher $d^{\prime}$ autres cellules de fusionner avec lui. Un tel système entraîne un gaspillage considérable. Toute union entre plus de deux gamètes donnera un zygote stérile: la méiose ne pourra pas résoudre l'augmentation de ploïdie causée par les fusions successives.

Dans un système monoïque, les cellules végétatives ont toutes le même génotype (figure 4). Elles se convertissent en gamètes de phénotypes (+) ou (-). Il s'agit d'une différenciation, puisqu'une même information génétique est utilisée de deux manières. On peut imaginer qu'un gène déterminateur (d) produit par épissage optionnel deux protéines distinctes, capables d'engager les cellules dans l'une ou l'autre voie de 
différenciation. Chez la drosophile, un tel phénomène intervient dans l'exécution par les cellules somatiques du programme de différenciation mâle ou femelle [23].

Les deux systèmes présentés (figure 4) fonctionnent aussi bien, que la gamétogenèse se déroule pendant la phase haploïde ou pendant la phase diploïde du cycle vital (figure 1). Dans un système dioïque, il suffit, pour produire des cellules diploïdes de deux phénotypes distincts, que le gène déterminateur existe sous deux formes: dominant/récessif, actif/ inactif ou présent/absent. Quant au système monoïque, il opère de la même façon suivant que les cellules contiennent une ou deux copies du gène déterminateur.

\section{Origine \\ des systèmes monoique et dioïque}

Les systèmes monoïque et dioïque coexistent dans les grands groupes d'organismes unicellulaires et multicellulaires: protistes, champignons, plantes vertes et métazoaires [7, 9, 24]. Chez les protistes coloniaux, les champignons et les ptéridophytes, la monoécie est connue sous le nom d'homothallie* [9]. Chez les métazoaires, la monoécie se traduit par l'hermaphrodisme. Parmi les protistes (ainsi que les champignons et les plantes supérieures), on trouve des espèces monoïques (homothalles) et dioïques (hétérothalles) qui sont étroitement apparentées [7, 9, 17]. Par ailleurs, les haplontes et les diplontes se partagent entre espèces monoïques et espèces dioïques [7, 9]. De cette diversité nous concluons deux choses. Premièrement, plusieurs changements de système ont eu lieu au cours de l'évolution des organismes unicellulaires et multicellulaires. Deuxièmement, aucun système ne possède un grand avantage sélectif vis-à-vis de l'autre.

\footnotetext{
* Chr les protistes coloniaux, monoécie el homothallie ne sont pas synonymes. Par exemple, un protiste colonial peut être $\dot{a}$ la fois homothalle el dioïque: un zygole donne naissance i des communaulés cellulaires de même génotype, qui produiront les unes des gamètes mâles, les autres des gamètes femelles. IJans ce cas, l'homothallie se réfere au génotype, tandis que la dioécie se réfore au phénotype. $\mathrm{m} / \mathrm{s} n^{\circ} 12$ vol. 9, dérembre 93
}

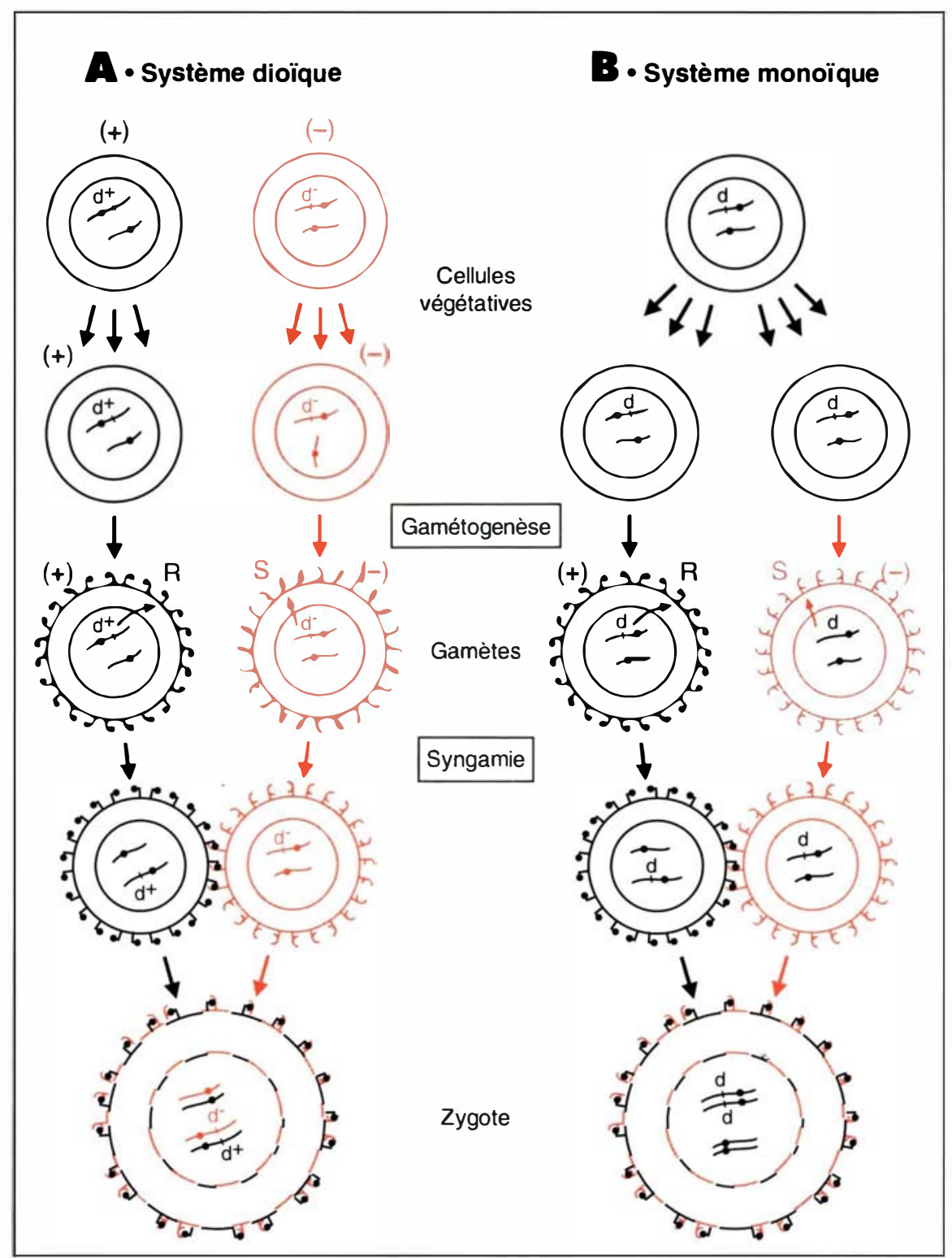

Figure 4. Fusion entre gamètes différents. Pour fonctionner correctement, la syngamie doit réunir des gamètes de phénotype différent. Les protistes satisfont à cette exigence en réalisant deux formes de gamétogenèse : dioïque ou monoïque. On suppose que l'une et l'autre se déroulent durant la phase haploïde du cycle vital. Un système dioïque idéal (A) fait intervenir deux catégories de cellules (+ ou -), différant par leur génotype. La gamétogenèse met en activité un ensemble de gènes qui gouvernent l'aptitude à fusionner. L'un de ces gènes (d) joue un rôle crucial dans le processus qui conduit à l'union des gamètes. II existe sous deux formes alléliques $\left(d^{+}\right.$ou d.). Sa fonction est de faire apparaître à la surface des gamètes (+) ou (-) des molécules complémentaires ( $R$ ou $S)$, permettant la reconnaissance mutuelle. Réunies dans le zygote, les molécules $R$ et $S$ rendent celui-ci inapte à toute nouvelle fusion. Un système monö̈que (B) ne comporte que des cellules de même génotype. Toutes possèdent la même forme du gène $d$. Pour se différencier en gamètes (+) ou (-), les cellules font un choix entre deux modes d'expression du gène $d$. Par exemple, le gène $d$ subit un épissage optionnel. L'un ou l'autre produit de l'épissage fait acquérir aux gamètes des molécules de reconnaissance complémentaires ( $R$ ou $S)$. Dans les modèles présentés, le gène $d$ peut, soit contrôler une étape clé dans la synthèse des molécules de reconnaissance, soit gouverner l'ensemble des gènes impliqués dans la gamétogenèse. Dans ce dernier cas, il s'agit d'un maître gène. 
Quel système apparut le premier au cours de l'évolution? Il nous semble que c'est la dioécie, en raison de sa simplicité (figure 4). Mais cet argument ne paraît pas très puissant. En tout cas, il est certain qu'en matière de détermination des types sexuels, le contrôle monogénique a connu un grand succès. Chez les protistes dioïques et monoïques, la détermination des types sexuels s'explique en général par l'action d'un seul gène qui existe sous deux formes ou s'exprime de deux façons [7]. Chez certains animaux, c'est la présence ou l'absence d'un gène qui détermine le sexe. C'est le cas pour les mammifères, où le gène déterminateur est un facteur de masculinité porté par le chromosome Y. I.e gène de masculinité humain (SRY) a été cloné récemment [25].

\section{Le chimiotropisme}

Depuis le début de la reproduction sexuée, la recherche d'un partenaire est une étape cruciale, préalable indispensable à la syngamie ou à la conjugaison. Afin d'accroître les chances de rencontre, les protistes ont développé un chimiotropisme analogue à celui qui permet aux bactéries de se diriger vers les sources de nourriture. Les cellules s'attirent en sécrétant une substance diffusible, qui est reconnue par un récepteur membranaire. Ce type de signal est appelé phéromone ou phérormone sexuelle.

Un tel mécanisme ne peut pas fonctiommer correctement s'il n'y a qu'une seule catégorie de gamètes, possédant les mêmes gènes gouvernant l'attraction réciproque. Si tous les gamètes sécrètent la même hormone et possèdent le même récepteur pour celle-ci, ils se paralysent car ils sont incapables de répondre à des facteurs diffusibles émanant d'autres cellules: leur propre récepteur est en permanence occupé par l'hormone qu'ils produisent. C'est le système autocrine bien connu des cancérologues, où une cellule tumorale stimule sa propre prolifération par un facteur de croissance qu'elle sécrète elle-même [26].

Le mécanisme d'attraction est viable s'il existe deux types de gamètes. L.es uns (-) émettent la substance attractive, tandis que les autres (+) y répondent [27]. Il est probable que le chimiotropisme apparut au cours de l'évolution des protistes plus tardivement que la différenciation des gamètes en deux types. Peu importe le mode de détermination des types sexuels adopté par les protistes archaiques (figure 4). Dans les deux cas, une liaison dut s'établir entre le gène déterminateur (d) et ceux qui gouvernent la synthèse de chaque élément du système chimiotrope, servant à la production et à la réception du signal attractif.

Rares sont les protistes che\% lesquels le tropisme gamétique a pu être démontré de façon convaincante. C'est le cas pour plusieurs espèces de sporozoaires [7] et de phytoflagellés, comme Chlamydomonas [28, 29]. Le chimiotropisme est employé par certaines algues et certains champignons $[28,29]$, ainsi que par divers animaux appartenant aux groupes des hydraires, des mollusques, des échinodermes et des urochordés [30].

\section{L'anisogamie}

Il est probable que che\% les protistes primitifs, la gamétogenèse était réduite à sa plus simple expression. L.es cellules végétatives se différenciaient en deux types, sans changer de morphologie. Elles devenaient seulement aptes à se recomnaître et à fusionner (figure 4). C.es modifications étaient déclenchées par une dégradation dans les conditions du milieu. C'est sans doute le stress causé par ces changements qui activait les gènes gouvernant le système de reconnaissance mutuelle.

Une gamétogenèse rudimentaire s'observe chez certains protistes : un gamète se forme à partir d'une cellule végétative [7]. L.es cellules sexuelles sont identiques, à l'exception des molécules membranaires qui gouvernent la recomnaissance réciproque. Dans ce cas, on parle d'isogamie, puisque les gamètes sont de taille et de mobilité égales, bien qu'ils appartiennent apparemment à deux types distincts (+ et -) [7]. Cette forme de gamétogenèse existe chez certains phytoflagellés et foraminifères [22]. On la considère en général comme primitive.
De nombreux protistes sont anisogames: ils produisent des gamètes de tailles inégales. Ia différence peut être minime. Elle est souvent considérable et conduit à la formation de deux catégories de cellules. L.es unes sont de petite taille et se déplacent en général grâce à un flagelle. I.es autres sont de grande taille, immobiles et remplies de matériaux de réserve. Il s'agit de spermatozoïdes et d'ovules, dont l'union est une véritable fécondation. On trouve des exemples d'anisogamie extrême che\% les sporozoaires et les phytoflagellés [22]. L'anisogamie est de règle chez les métazoaires.

Pour expliquer l'émergence de l'anisogamie, une théorie séduisante [31] oppose deux pressions sélectives opposées. A partir d'une masse donnée de matière vivante, il vaut mieux produire davantage de gamètes de petite taille parce qu'ils peuvent être émis en plus grand nombre. Supposons par ailleurs que la viabilité du zygote augmente plus qu'en proportion de son volume. Pour l'organisme, il sera avantageux de produire de gros gamètes parce qu'ils donnent naissance à des zygotes de grande taille, qui résisteront mieux aux conditions défavorables. Les gamètes de dimensions intermédiaires seront moins aptes, donc défavorisés. Ainsi se crée une sélection "disruptive" sur la taille des gamètes, engendrant l'anisogamie. C.e raisonnement peut être formulé sous une forme légèrement différente: il est difficile pour un gamète d'être performant à la fois sur le plan de la mobilité et sur celui de l'accumulation des réserves, d'où la sélection "disruptive " déjà évoquée. Nous considérons que la sélection disruptive a joué d'abord non sur la taille des gamètes, mais sur leur mobilité. C'est probablement l'acquisition du chimiotropisme qui engendra cette différence de comportement. Si les cellules s'attirent, elles ne tardent pas à se différencier en deux catégories, les unes rapides, les autres lentes [27, 32]. L.es cellules rapides sont celles qui répondent au signal hormonal. Elles rencontrent plus facilement les cellules lentes, qui émettent le signal. De leur côté, les cellules lentes agrandissent l'espace où s'exerce l'action de $\mathrm{m} / \mathrm{s} n^{\circ} 12 \mathrm{vol}$. 9, decembre 93 
l'hormone qu'elles émettent. Elles accroissent ainsi leur pouvoir attractif [32]. De fait, certains flagellés apparemment isogames s'immobilisent quand ils commencent à attirer leurs congénères [28, 29].

Il est logique de penser qu'une différence de mobilité entre gamètes entraina à son tour un dimorphisme de taille. Une petite cellule étant plus facile à mouvoir qu'une grande, on conçoit que l'évolution ait favorisé les mutations tendant à réduire la taille des gamètes rapides. Elle dut aussi retenir les mutations qui ont pour effet d'augmenter le volume des gamètes lents. Cet agrandissement a deux avantages. Il ralentit la vitesse de déplacement des cellules, ce qui élargit l'espace où agit le signal attractif qu'elles émettent [32]. Il accroît aussi les chances de survie du zygote. Par le jeu d'une double pression sélective sur la taille et la mobilité des gamètes, le dimorphisme sexuel doit s'accentuer peu à peu. Le dimorphisme est effectivement considérable che\% beaucoup de protistes et chez. les métazoaires.

\section{Les étapes conduisant à l'anisogamie}

Che\% les protistes, l'anisogamie peut être considérée comme le stade ultime d'une longue évolution. Cette tendance évolutive paraît irréversible. En effet, il semble impossible pour une espèce anisogame de revenir à l'isogamie. Nous distinguons quatre étapes principales dans l'acquisition de l'anisogamie : diploïdisation, différenciation en deux types sexuels, apparition du chimiotropisme, apparition du dimorphisme gamétique (figure 5).

Suivant le schéma proposé, la diploïdisation aurait engendré des espèces homogames, qui produisaient une seule sorte de gamètes (figure 3). De telles espèces n'ont peut-être jamais existé. A notre commaissance, aucune n'a été découverte à l'heure actuelle. Il subsiste peu d'espèces isogames produisant des gamètes mobiles (figure 5). On les trouve parmi les phytoflagellés et les foraminifères [7]. Ces espèces seraient les témoins d'un stade évolutif antérieur à l'adoption du chimiotro$\mathrm{m} / \mathrm{s} \quad 1^{\circ} 12 \mathrm{vol}$. 9, décembre 93 pisme. Il n'y a apparemment aucun protiste dont les gamètes soient à la fois de même taille, mobiles et capables de s'attirer [28]. C'est sans doute parce que le chimiotropisme fit rapidement disparaitre l'isogamie [27, 32]. On a décrit quelques espèces isogames dont les gamètes s'immobilisent quand ils attirent leurs congénères demeurés mobiles: Chlamydomonas paupera; C. moenusii $[28,29]$. Nous les considérons comme des vestiges d'une évolution arrêtée sur la voie de l'anisogamie. L.e schéma évolutif présenté accorde un rôle important au chimiotropisme. Toutefois, ce phénomène n'a pu être mis en évidence que dans un nombre restreint d'espèces anisogames [28-30]. On sait maintenant que le chimiotropisme est plus répandu qu'on ne le croyait il y a quelques années [28], parce qu'il est difficile à démontrer [30]. Néanmoins, il est probable que le chimiotropisme a disparu dans certaines lignées. Une cause possible est l'accentuation de l'anisogamie. Devenant plus nombreux et plus agiles, les spermatozoïdes trouvent plus facilement leur cible, sans que celleci ait à les attirer.

\section{Nombre de types sexuels}

Lors d'une syngamie normale, deux cellules fusiomment pour former un zygote. Toutefois, le nombre de types sexuels n'est pas nécessairement limité à deux. Pour certains auteurs, ce nombre est théoriquement illimité [33]. Les types sexuels sont souvent nombreux che\% les champignons [34]. L.e myxomycète Physarum produit plus de 10 types d'isogamètes, capables de fusionner deux à deux [35]. Il y a quelque utilité à multiplier les types sexuels. Dans un système binaire, un gamète ne peut s'unir qu'à un seul type de congénère. Quand les types sexuels se multiplient, le choix s'élargit: un gamète est autorisé à fusiomner avec des congénères de plusieurs types différents. On dit qu'il bénéficie de "l'avantage du rare".

Malgré cet avantage, le système à types sexuels multiples ne s'est pas généralisé. Che\% les protistes, ce système semble limité aux ciliés [7].
Ceux-ci emploient divers moyens pour accroître le nombre de types sexuels. Les espèces diö̈ques font dépendre de plusieurs gènes la détermination des types sexuels, ou multiplient les allèles du gène déterminateur. Les espèces monoïques utilisent plus de deux modes de différenciation $[7,18,36]$.

Comment expliquer la prépondérance du système binaire? Une théorie récente invoque une incompatibilité entre les génomes cytoplasmiques apportés au zygote par les mitochondries ou les chloroplastes des gamètes [35]. Ce modèle prévoit que c'est un gène nucléaire qui résout le conflit entre les gènes cytoplasmiques. Un allèle de ce gène rend les organites de la cellule (-) incapables de détruire ceux de la cellule (+), mais vulnérables à l'attaque de ces derniers. Cet allèle définit le sexe mâle: c'est celui qui ne transmet pas ses gènes cytoplasmiques à la génération suivante. Le modèle permet effectivement de comprendre la multiplication des types sexuels chez les ciliés car ceuxci n'échangent pas leurs gènes cytoplasmiques durant la conjugaison.

En ce qui concerne les espèces pratiquant la syngamie, la théorie nous semble moins pertinente. Si le système bisexuel tire vraiment son origine d'un combat entre organites, il faut s'attendre que le conflit intracellulaire persiste che\% les organismes actuels. Il s'ensuit que dans les espèces bisexuées, l'hérédité de l'ADN cytoplasmique devrait être uniparentale. C'est le cas pour le phytoflagellé Chlamydomonas: les deux gamètes fournissent au zygote un lot d'ADN chloroplastique, mais celui du gamète (-) est détruit, apparemment par une endonucléase de restriction contenue dans le chloroplaste de la cellule (+) [37].

Il serait intéressant d'étudier l'hérédité des gènes cytoplasmiques dans d'autres espèces de protistes, afin de voir si le conflit des génomes est aussi répandu que la théorie le prévoit. Chez les métazoaires, le zygote ne reçoit son $\mathrm{ADN}$ mitochondrial que du gamète femelle. Mais cette règle n'est pas absolue. Che\% la moule par exemple, l'ADN mitochondrial se transmet par le spermatoroïde et par l'ovule [38]. De 


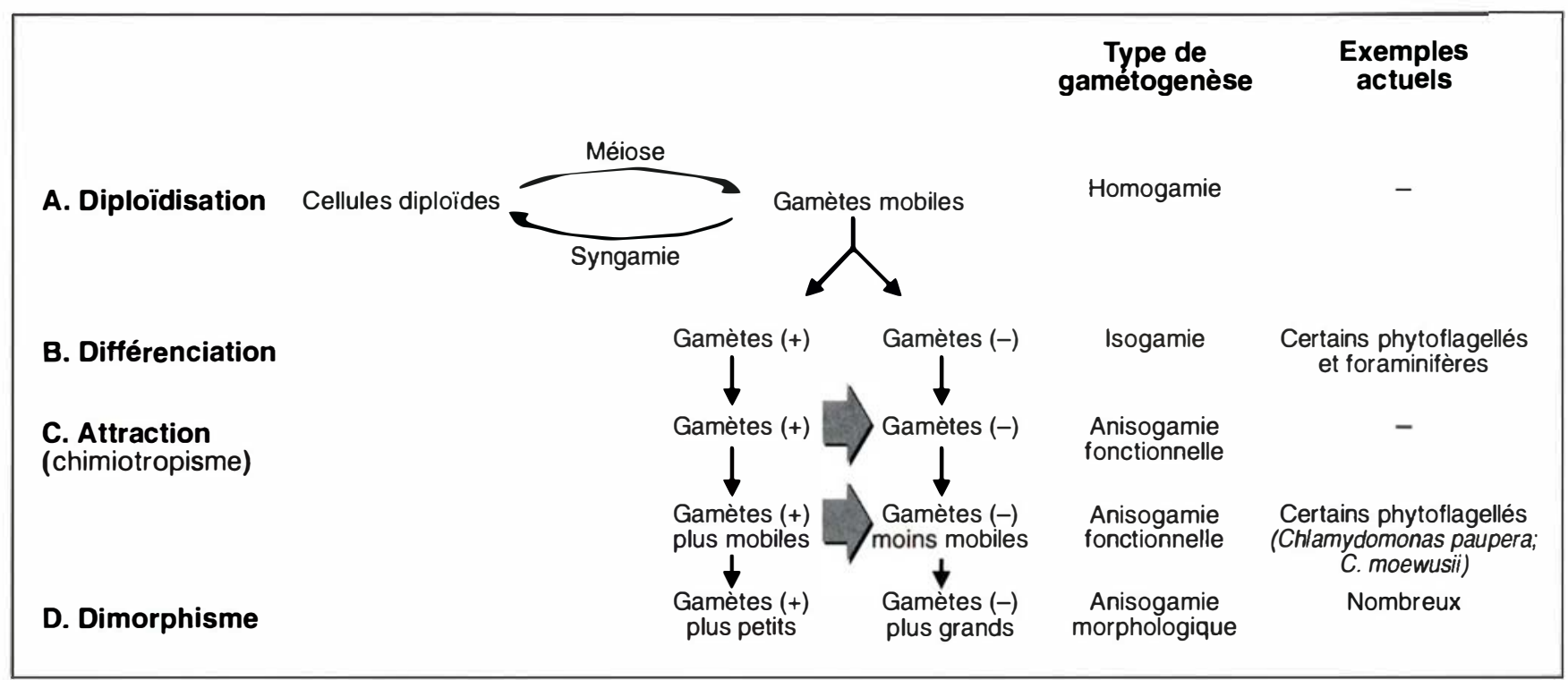

Figure 5. Acquisition de l'anisogamie par les protistes. Le schéma proposé fait intervenir quatre événements essentiels. On ne peut pas préciser si chacun d'eux se produisit une seule fois ou plusieurs fois au cours de l'évolution. Le premier événement (A) est la diploïdisation. II implique deux acquisitions majeures: la syngamie, qui instaure la phase diploïde, et la méiose, qui rétablit l'haploïdie. Le deuxième événement (B) est la différenciation des gamètes en deux types (+ ou -). Le troisième événement (C) est l'apparition d'un pouvoir attractif entre gamètes. Cela entraîna l'émergence d'un dimorphisme gamétique, d'abord en ce qui concerne la mobilité, ensuite en ce qui concerne la taille des cellules (D). La plupart des espèces actuelles sont anisogames, mais seule une petite minorité d'entre elles pratique le chimiotropisme.

même, le spermatozoïde de drosophile et de souris lègue à l'œuf au moins une partie de son ADN mitor chondrial [39, 40].

Il est peut-être inutile d'invoquer une hypothétique guerre intestine pour expliquer pourquoi le système bisexuel s'est imposé presque partout. Si cette guerre a réellement joué un rôle, celui-ci n'a pu concerner qu'une étape évolutive bien délimitée, qui précéda l'acquisition de l'anisogamie. Il est probable que l'anisogamie apparut chez des protistes où le nombre de types sexuels était limité à deux (figure 5). En effet, on voit mal comment un dimorphisme gamétique aurait pu s'instaurer dans une lignée à types sexuels multiples. Une fois l'anisogamie acquise, il devenait impossible de multiplier les types sexuels. Une espèce anisogame est liée au système binaire: elle ne peut produire que deux sortes de gamètes.

\section{L'héritage et les innovations des métazoaires}

Deux théories ont été proposées pour expliquer l'apparition des métazoaires. La théorie coloniale envisage une réunion en agrégats de cellules isolées, qui seraient des flagellés [22]. Une autre théorie fait dériver les métazoaires de cellules plurinucléées, qui seraient des ciliés [41]. Des événements indépendants pourraient être à l'origine des autres formes supérieures de vie (champignons et plantes vertes). Les deux théories supposent que les prédécesseurs directs des animaux étaient des diplontes. Cela signifie que chez les ancêtres des métazoaires, la méiose se produisait à la fin de la phase diploïde (figure 1).

Quels autres caractères furent transmis par les protozoaires aux premiers métazoaires? Il est certain que la méiose de type 2 était incluse dans l'héritage. Nous pensons que l'anisogamie faisait aussi partie des caractères hérités, sous la forme de gènes commandant la transformation des cellules sexuelles en spermatozoïdes ou en ovules. Il est difficile de dire si les ancêtres directs des métazoaires étaient dioïques ou monoïques. En effet, l'hermaphrodisme et le gonochorisme coexistent dans de nombreux groupes zoologiques [24].
Du point de vue reproductif, les animaux firent deux grandes innovations. La première fut de confier la reproduction à une catégorie particulière de cellules, qui forme le germen [15]. La seconde innovation fut d'inclure les cellules germinales dans des organes spécialisés, dérivant du soma : les ovaires ou les testicules [42]. Une conséquence importante découle de ces acquisitions : c'est le soma qui aiguille les cellules germinales dans la voie de différenciation mâle ou femelle [42]. Chez les espèces hermaphrodites, le soma d'un même individu transforme en ovules ou en spermatozoïdes des cellules germinales de même génotype. Mais l'hermaphrodisme peut aussi se manifester dans des espèces où le sexe est déterminé génétiquement. Des facteurs épigénétiques, comme la température, premnent alors le pas sur les facteurs génétiques. Cela se produit notamment chez certains amphibiens et reptiles [43].

\section{La méiose} des métazoaires femelles

Les métazoaires ont poussé jusqu'à 
l'extrême l'anisogamie déjà développée par les protistes. Cette tendance évolutive nécessita de nombreuses adaptations, qui concernent principalement l'ovogenèse. En effet, il est difficile de produire des ovules de très grande taille. L'obstacle principal consiste en l'élaboration par l'ovocyte de réserves abondantes d'ARN et de protéines. C'est particulièrement vrai en ce qui concerne l'ARN, et ce pour deux raisons. D'abord, l'ARN ne traverse pas les barrières membranaires. Ensuite, sa vitesse de synthèse est limitée par le nombre de copies de gènes disponibles. Pour surmonter cette difficulté, les métazoaires ont adopté des solutions très diverses. Parmi celles-ci, deux sont communes à tous les groupes zoologiques. Il est donc probable que leur apparition remonte à une période très précoce de l'évolution animale. Il s'agit de modifications de la méiose.

La durée de la méiose est allongée chez les femelles, ce qui donne à l'ovocyte du temps pour s'accroître. Il y a entre le début et la fin de la méiose un intervalle qui, suivant les espèces, se compte en semaines, en mois, voire en années. L'allongement concerne la prophase de la première division méiotique. Cela fait que, pendant toute la durée de son accroissement, l'ovocyte contient quatre assortiments de chromosomes (figure 2). Par rapport à la cellule haploïde qu'il va engendrer, l'ovocyte possède quatre fois plus d'ADN, donc une capacité de production d'ARN quadruplée. Cet avantage n'est pas négligeable.

Chez les femelles, la méiose se termine par la production non pas de quatre cellules de taille égale, comme dans la spermatogenèse, mais d'une seule cellule fonctionnelle : l'ovule. Les trois autres compléments de chromosomes sont éliminés avec un minimum de cytoplasme, sous la forme de globules polaires. L'ovule conserve donc pour lui seul presque tout ce que l'ovocyte a accumulé pendant son accroissement. Un phénomène analogue s'observe pendant la gamétogenèse de certains protistes appartenant au groupe des héliozoaires [17]. Chaque division méiotique engendre deux noyaux, mais l'un d'eux dégénère. La méiose ne produit donc qu'un seul gamète haploïde. Dans plusieurs espèces de volvocales (qui sont des haplontes), une seule cellule émerge également de la méiose, mais c'est après la formation du zygote qu'a lieu l'élimination des noyaux excédentaires [ 7 , 8].

\section{La division du travail}

Les métazoaires utilisent différents moyens pour rendre l'ovogenèse plus efficace. Cela leur permet de produire en moins de temps des ovocytes plus gros. Le procédé le plus archaïque est employé par les animaux didermiques: éponges et cnidaires, dont les gonades sont inexistantes ou rudimentaires [42]. Pour s'accroître, les ovocytes capturent et phagocytent d'autres cellules que l'on appelle nourricières $[44,45]$.

Chez les cténaires et les animaux tridermiques, les ovocytes sont enfermés dans un ovaire [42]. La coopération cellulaire ne fait plus intervenir la phagocytose, mais implique des cellules éloignées des gonades. C'est ce qui se produit pour les protéines du vitellus. Dans de nombreux groupes zoologiques (nématodes, crustacés, insectes, échinodermes, vertébrés), on a montré que ces protéines sont synthétisées par des cellules somatiques, véhiculées par les fluides corporels, puis absorbées par les ovocytes [42].

Les protéines peuvent être assez facilement transportées de cellule à cellule. Il n'en va pas de même pour l'ARN. Pour couvrir leurs besoins en ARN, les ovocytes recourent à des solutions particulières. Dans plusieurs groupes zoologiques, les ovocytes renforcent leur capacité de production pour un ARN déterminé. Il peut s'agir d'une amplification (multiplication) des gènes spécifiant les ARN ribosomiques $28 \mathrm{~S}$ et 18S, comme cela se produit chez les amphibiens, les téléostéens et certains insectes [46]. Il peut aussi s'agir de la mise en activité d'un lot de gènes réservé à l'usage exclusif de l'ovocyte. Cette stratégie est utilisée par les amphibiens et les téléostéens pour la production d'ARN ribosomique 5S [47].

Une solution originale consiste à multiplier les noyaux dans l'ovocyte. Elle a été adoptée par certains amphibiens tels que la grenouille à pénis Ascaphus et les grenouilles marsupiales [48, 49]. Les ovogonies de ces animaux subissent plusieurs divisions nucléaires (3 à 11), sans que leur cytoplasme se segmente. Les ovocytes en accroissement contiennent 8 à environ 2000 vésicules germinatives (noyaux), ce qui accroît d'autant leur capacité de production d'ARN. A la fin de l'ovogenèse, il subsiste un seul noyau, qui achèvera la méiose.

Chez de nombreux insectes, le partage du travail se fait comme chez Ascaphus, à ceci près que la coopération fait le plus souvent intervenir des cellules au lieu de noyaux. Les ovogonies se divisent plusieurs fois. Les caryocinèses sont suivies de cytocinèses incomplètes, si bien que les cellules filles restent en communication par leur cytoplasme [50]. Un choix se fait parmi les cellules d'un même clone. L'une devient ovocyte. Toutes les autres deviennent nourricières et se vident de leur contenu au profit de l'ovocyte, qui grossit rapidement. Une situation extrême se rencontre chez les hémiptères et certains coléoptères : plusieurs ovocytes sont reliés par un cordon nourricier à un syncytium réunissant un grand nombre de noyaux [50]. Grâce à la continuité cytoplasmique, l'ovocyte absorbe l'ARN ainsi que les autres molécules et organites élaborés par la communauté.

Il existe des cellules nourricières dans plusieurs groupes d'invertébrés : cténaires, annélides, myriapodes, crustacés, échinodermes [50, 51]. Comme chez les insectes, il semble que les cellules nourricières soient d'origine germinale. Il arrive même qu'une spécialisation de l'ovaire favorise la nutrition des ovocytes. C'est ainsi que chez les rotifères, la plus grande partie de l'ovaire (le vitellogène) élabore les substances nutritives qu'absorbent les ovocytes formés dans une autre région de la glande génitale (le germigène) [52].

\section{Conclusion}

Beaucoup de biologistes se sont étonnés que la reproduction sexuée 
ait pu apparaître, puis se maintenir face à d'autres modes de propagation, comme la multiplication végétative et la reproduction asexuée, qui sont apparemment plus efficaces. La reproduction sexuée a un avantage évident : elle entretient de la diversité parmi les organismes qui la pratiquent, en créant de nouvelles associations de gènes grâce à la méiose et à la recombinaison [53]. La diversité génétique offre une large prise à la sélection naturelle, qui favorisera les ineilleures adaptations au milieu. Mais la reproduction sexuée a aussi de nombreux inconvénients. Parmi ceux qui ont été recensés, il en est un qui affecte tous les organismes pratiquant ce mode de propagation: la recherche d'un partenaire sexuel est coûteuse en temps et en énergie. Elle est donc susceptible de ralentir la multiplication des individus [53].

Il est difficile de peser les avantages et les inconvénients que procure la reproduction sexuée. C'est à court terme que les inconvénients se manifest ent. C.e n'est qu'à très long terme que peuvent apparaitre les effets positifs, dont le principal sera l'émergence des formes de vie les plus complexes. Si l'on poursuit cette réflexion, on est tenté d'attribuer quelque valeur prédictive à la reproduction sexuée, puisqu'elle apparut bien avant que les organismes aient pu en tirer tous les bénéfices. Mais ce type de raisonnement est fallacieux. En matière d'évolution, les prémonitions n'existent pas. Seuls jouent les causes et les effets immédiats, qu'il faut s'efforcer de distinguer des conséquences lointaines.

En ce qui concerne l'émergence de la reproduction sexuée, nous suggérons simplement que, dès le début, ce mode de propagation donna aux organismes qui l'inventèrent un certain avantage par rapport aux autres. Cela paraît plausible si l'on admet que la reproduction sexuée fut, à l'origine, une adaptation à un enviromement hostile. De telles circonstances abolissaient une compétition fondée sur la vitesse de multiplication. Pour les cellules, l'enjeu à court terme était de rester en vie jusqu'au retour des conditions favorables. A ce stade, une supériorité même minime pouvait donner une avance décisive pour la conquête du milieu

\section{Remerciements}

Les auteurs remercient Janine Beisson, Jean Génermont, Bernard Godelle, Jean-Claude lacroix, Hervé le Guyader, Jean-Pierre Mignot et Maurice W'egnez pour leurs critiques et leurs conseils pendant la préparation du manuscrit de cet article.

\section{Herman Denis}

Professeur à l'université Pierre-et-MarieCurie.

Alain Collenot

Professeur retraité de l'université Pierre-et-Marie-Curie.

Centre de génétique moléculaire, Cnrs, avenue de la Terrasse, 91198 Gif-surYvette Cedex, France.

\section{RÉFÉRENCES}

1. Jacob F. I jeu des prossibles. Paris: Favard, 1981.

2. Mavnard-Smith J. The erolution of sex. Cambridge: (Cambridge L'niversity Press, 1978 .

3. Margulis L, Sagan D. (Mrigins of sex: three billiom years of genetic recombination. New Haven : Yale L'niversin Press, 1986.

4. Bell (;. The masterpiece of nature: the evolution and genetics of sexuality. Berkeley: University of California Press, 1982.

5. Margulis I, Sagan D, Olendzenski I. What is sex? In: Halvorson HO, Monroy $\mathrm{A}$, eds. The origin and evolution of sex. New York: Liss, 1985: 69-85.

6. Raikov IB. The Protozoan Nucleus. Morphology and Evolution. Viemne: Springer, 1982.

7. Grell K(;. I'rotozoology). Berlin : Springer, 1973.

8. Mignot JP. Les coenobes chez les volvocales: un exemple de passage des unicellulaires aux pluricellulaires. Ann Biol $1985: 24: 1-26$.

9. Plantefol L. Cours de botanique el de biologie végétale. Paris: Belin, 1950.

10. Shigenaka Y, Kaneda M. Studies on the cell fusion of Heliozoans IV. An electron microscopical study on the fusion process accompanied with axopodial degradation. Annol Zool Jpn 1979; 52: 28-39.

11. Goodenough UW. An assay on the origins and evolution of eukaryotic sex. In: Halvorson $\mathrm{HO}, \mathrm{Monroy} \mathrm{A}$, eds. The origin and evolution of sex. New York: Liss, 1985 : 123-40.
12. Cleveland IR. Hormone-induced sexual cycles of flagellates VII. One-division meiosis and autogamy without cell division in Urinympha. J Morphol 1951 : 88 : 385-439

13. Hurst I.D, Nurse P. A note on the evolution of meiosis. J Theor Biol 1991; 15(): 561-3.

14. Hollande A, Carruette-Valentin J. Appariement chromosomique et complexes synaptonématiques dans les noyaux en cours de dépolyplö̈disation che Rrsonympha flagellata: le cycle évolutif des Pyosonymphines symbiontes de Reticulitermes lucifugus. ('TR Acad Sir P'aris 197(); 27() : 25.5()$-3$.

15. Denis H, I acroix, JC. I'origine de la lignée germinale et de la mortalité cellulaire. médecine/sciences 1992; 8: 695-7()2

16. Sinchen (;, Hugerat Y'. W'hat determines whether chromosomes segregate reductionally or equationally in meiosis? Bio lissajs 1993; 15: 1-8.

17. Beam C.A, Himes M. Sexualio and meiosis in dinoflagellates. In : I cevandowsky M. Hutner SH, eds. Biocheonistry and physiology' of Prolozoa. Volume 3. New York: Academic Press, 198(): 171-206.

18. de Puvtorac P, Grain J, Mignot JP. Pré cis de protistologie. Paris: Boubée, 1987.

19. Hawley RS, Arbel T. Yeast genetics and the fall of the classical view of meiosis. Cell $1993 ; 72: 3() 1-3$.

2(). Molon-Noblot S, Dosportes 1. Mise en évidence de complexes synaptonématiques dans le norau méiotique d'un Sporozoaire la Grégarine Cirflmickiella gracilis (Crebnicki) parasite de la Scolopendre Scolopendra cingulata I. (IR Acal Sor Paris 1977 . 285: $217-9$

21. Hartwell IH, Weinert TA. Checkpoints: controls that ensure the order of cell cycle events. Srience 1989; 246: 629-34.

22. Hyman I.H. The Invertebrates: Protozoa through Cienophora. New York: McCraw-Hill, 194() .

23. Dargouge O. I.es cascades du sexe che\% la mouche. La Recherche 1989 ; 2() : 153()-2

24. Gassé PP, Poisson RA, Tuzet O. Zoologie. Tome i. Invertébrés. Paris: Masson, 1961

25. Sinclair AH, Berta P, Palmer MS, ot al. A gene from the human sex-determining region encodes a protein with homology to a conserved DNA-binding motif. Nature 199()$; 346: 24()-4$

26. Sporn MB, Todaro (JJ. Autocrine secretion and malignant transformation of cells $N$ lingl / Med 198(); 3()3: 878-8().

27. Hoekstra RF. Evolution of gamete motility differences II. Interaction with the evolution of anisogamy. I Theor Biol 1984 $107: 71-83$.

28. Machlis I, Rawitscher-Kunkel E. Mechanisms of gametic approach in plants. Annu Rev Cytol 1963; 15: 97-138.

29. Kochert (;. Sexual pheromones in algae and fungi. Annu Rnt Plant Physiol 1978; 29 : $461-86$. 
3(). Miller RI.. Sperm chemo-orientation in the Metazod. In: Met\% (.BB, Momroy A, eds. Biology of fertilization, vol 1. ()rlando: Academic Press, 1985: 275-337

31. Parker (iA, Baker RR, Smith V(iF. The origin and evolution of gamete dimorphism and the male-female phenomenom. / Theor Biol 1972; 36: 529-53.

32. Hockstra RF, Jamy RF, Schilstra AJ. Frolution of gametc mobility differences I Relation between swimming speed and pheromonal attraction. I Theor Biol 1984; $107: 57-7()$.

33. Iwasa Y, Sasaki A. Fvolution of the number of sexes. Evolution 1987; 41 : 49-(i.).

34. Fincham JRS, Day PR. Irungal gemelic. ()xford: Blackwell, 1963.

35. Hurst L.1), Hamilton W1). (vitoplasmic fusion and the nature of sexes. Pror $R$ So I.ond (Biol) 1992; 247: $189-94$.

36. Orias E Probable somatic DNA rearrangements in mating type determination in Tetrahymena thermofhila: a review and a model. Den (ipn 1981; 2): 185-202.

37. Sager R, Gabowr C. Sex in C.hamyrlomonas: sex and the single chloreplast. In Halvorson H(), Momroy A eds. The origin and enolution of sex. New York: I iss, 198: : $11.3-21$.

38. \%ouros E, freeman KR, Ball A(), Pog son (ilt. Direct evidence for extensive paternal mitonchondrial 1)NA inheritance in the marine mussel Mylilus. Nature 1992; $359: 412-4$

39. Kindo R, Satla Y, Matsuma ET, Ishiwa $\mathrm{H}$, Takahata $\mathrm{N}$, Chigusa SI. Incomplete maternal transmission of mitochondria 1)NA in Drosophila. (ienelies 1990); 126: (5.57-6.3.

40. (ivllensten l: Wharton 1), Josefisson A Wilsom AC. Paterual inheritance of mitochondrial 1)NA in mice. Nalure 1991; 352 2:5:)-7.

41. Hadri I. The erolution of Melazoa. New York: Pergamon Press, 1963.

42. Denis H, I acroix JC. Une interprétation érolutive de la gamétogenése animale. médecine/sciences 1993; 9: 752-61.

43. 1)ournon (:, Houillon (:, Pieau (. Temperature sex-reversal in amphibians and reptiles. Int / Dev Biol 199(); 34: 81-92.

44. Tuzet (O. I 'origine de la lignée germinale et la gamétogenèse chez les spongiaires. In: Wolff F, ed. L'origine de la lignée genminale Paris: Hermamm, 1964: 77-111. 45. Tardent P. The differentiation of germ cells in (nidaria. In : Halvorson HO, Monrov $A$, eds. The origin and erolution of sex New York: I.iss, 1985: 163-97.

46. Tobler H. Occurrence and developmental significance of gene amplification. In: Weber $\mathrm{R}$, ed. The biochernistry of animal develofment, vol III. New York: Academic Press, 1975: 91-143.

47. V'an den Eynde H, Marabraud A, Denis H. Biochemical research on oogenesis. RNA accumulation in the oocvies of the newt Plenirodeles anall. Derielopiment 1989; 105: $11-(6$

48. Macgregor HC:, Kezer J. ('ene amplification in oocrtes with 8 germinal vesicles from the tailed frog Ascaphus moe Stejueger. (:hromosoma 1970 ; 29 : $189-206$ 49. del Pino EM, Humphries AA. Multiple nuclei during early oogenesis in flectomo-

$\mathrm{m} / \mathrm{s} n^{\circ} 12$ vol. 9, déeembre 93 lus pygmaens and other marsupial frogs. Biol Bull 1978; 154: 198-212

5). Raven (.P. ()ogenesis: the storage of derolopmental information. ()xford: Pergamon, 1961.

51. Dunlap Pianka H. Citenophorat. ln: (iese AC:, Pearse JS, eds. Reprorluclion of marine imvertebrales, vol I. Acoelomates and pseudocoelomale melazoans. New York: Acatdemic Press, 1974: 2(0)-(6.5.

52. de Beauchamp P. Classe des rotifères. ln: (irassé PP, ed. Traile de zoologie, tome IV', fascicule III. Paris: Massom, 1965: $1225-379$

53. (iomon PH, Maturice $\mathrm{S}$, Reboud $X$, Till-Botiraud l. I.e sexe pour quoi faire? I.a Recherche 1993: 24: 7()-(i)

\section{Summary}

Origin and evolution of sexual reproduction

Sexually reproducing organisms have alternating haploid and diploid phases. Each phase is connected to the other through meiosis and fertilization or conjugation. This article provides a tentative explanation for several features of sexual reproduction, and in particular for anisogamy, i.e. ability to produce gametes of unequal sizes. Chemotropism may be regarded as the driving force which led to acquisition of anisogamy. A chemotropic system functions optimally if the gametes belong to two distinct categories. One category of cells is nonmotile and secretes an attractive substance, whilst the other category responds to the attractive signal by moving towards the emitting cells. Anisogamy is thought to result from selection of two types of mutations: those increasing the size of the nonmotile gametes, and those reducing the size of the motile gametes. Such modifications have clear advantages. Larger and slower cells increase their attractive power and give rise to zygotes with higher survival fitness. Smaller cells gain swimming speed and can be produced in larger numbers. This gives them a better chance of finding a partner to mate with.

\section{TIRÉS A PART}

\section{H. Denis.}

